\title{
Atomic Level Structure-Property Relationship in a Spin-Orbit Mott insulator: Scanning Transmission Electron and Scanning Tunneling Microscopy Studies
}

\author{
Maxim Ziatdinov ${ }^{1,2}$, Artem Maksov ${ }^{1,2}$, Wu Zhou ${ }^{3}$, Arthur Baddorf ${ }^{1,2}$, and Sergei Kalinin ${ }^{1,2}$ \\ 1. Institute for Functional Imaging of Materials, Oak Ridge National Laboratory, Oak Ridge TN 37831 \\ USA \\ 2. Center for Nanophase Materials Sciences, Oak Ridge National Laboratory, Oak Ridge TN 37831 USA \\ ${ }^{3}$ Materials Science and Technology Division, Oak Ridge National Laboratory, Oak Ridge TN 37831 \\ USA
}

Advances in scanning transmission electron microscopy (STEM) and scanning tunneling microscopy (STM) techniques have recently allowed researchers to measure materials structural and electronic properties, such as atomic displacements and charge density modulations, at a picometer scale in a real space. It is now realized that the full information recovery from high-resolution image datasets can be achieved by adopting the big-data analytical tools, such as multivariate statistical analysis and unsupervised machine learning [1]. Here we used multivariate analysis to study statistically significant variations in structural parameters and charge density patterns in a spin-orbit Mott insulator $\alpha-\mathrm{RuCl} 3$ [2].

The average STEM unit cell of $\alpha-\mathrm{RuCl}_{3}$ films [Fig. 1 (a)] showed a good agreement with neutron and X-Ray data analysis providing us a good understanding of a long range atomic order in the $\mathrm{RuCl}_{3}$ system. We then used an information contained in microscopic degrees of freedom available from STEM images to characterize atomic structure on a local scale. Particularly, we employ a principle component analysis (PCA) to search for statistically significant variations of an average unit cell structure [Fig. 1(b-c)]. This issue is important because the varying inter-column distances within the unit cell usually have powerful and non-random effects on the electronic structure of strongly-correlated systems. Interestingly, we found only relatively small displacements from average structure in the overlapped $\mathrm{Ru} / \mathrm{Cl}$ columns [Fig. 1(b)]. As the location of $\mathrm{Ru} / \mathrm{Cl}$ column centers is determined mainly by a contribution from the Ru core electrons, the results in Fig. 1(b) indicates that the positions of Ru atoms in a honeycomb lattice remain nearly fixed. On the other hand, we observed relatively large variations from average in the positions of $\mathrm{Cl}$ columns. This shows that intertwined structural order parameter fields in $\mathrm{RuCl}_{3}$ films occur mainly within the $\mathrm{Cl}$ subsystem.

Having analyzed in detail the local structural properties of $\alpha-\mathrm{RuCl}_{3}$ crystals, we turned to a realspace characterization of its electronic structure by virtue of low-bias STM imaging. To explore details of the real-space electronic behavior on a local scale, we first identified positions of electron density peaks associated with an underlying atomic lattice. For this purpose, we created a template unit cell from FFT-filtered image data, and then perform normalized cross-correlation analysis combined with position based and intensity based refinements in order to extract maximal electron densities associated with $\mathrm{Cl}-$ terminated surface lattice structure [Fig. 2(a)]. Once all the positions of electron density peaks are identified, the histogram-based visualization of electron density distribution reveals a presence of a peculiar charge ordered (CO) phase manifested as an intra-unit cell electronic symmetry breaking at the Cl-terminated surface [Fig. 2(b)]. Finally, we discuss a fine structure of the CO pattern, in which two coexisting electronic superlattices, rhombic and honeycomb, form distinctive clusters at a subnanometer length scale [Fig. 2(c)]. The peculiar switching between two surface electronic superlattices is explained by variations of an average unit cell structure in the $\mathrm{Cl}$ subsystem, found from analysis of STEM data, and the nanoscale strain fields associated with intertwined structural orders. 


\section{References:}

[1] S.V. Kalinin, et al., Nature Mater. 14 (2015), 973-980.

[2] M. Ziatdinov, to be submitted.

[3] This research was sponsored by the Division of Materials Sciences and Engineering, BES, DOE. Research was conducted at the Center for Nanophase Materials Sciences, which is a DOE Office of Science User Facility.

(a)

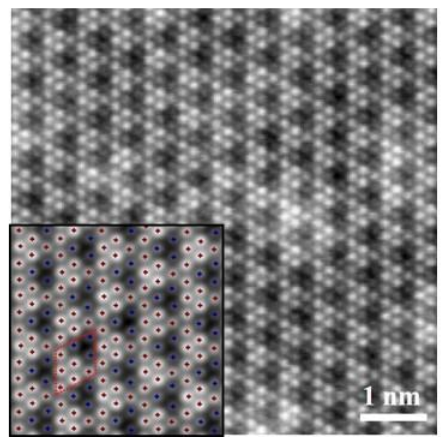

(b)

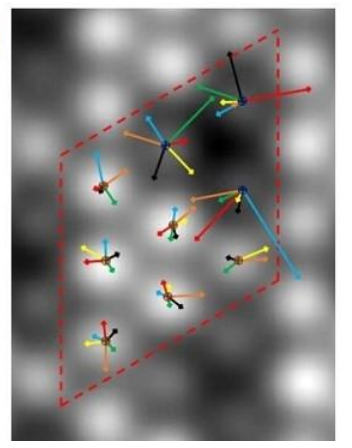

(c)

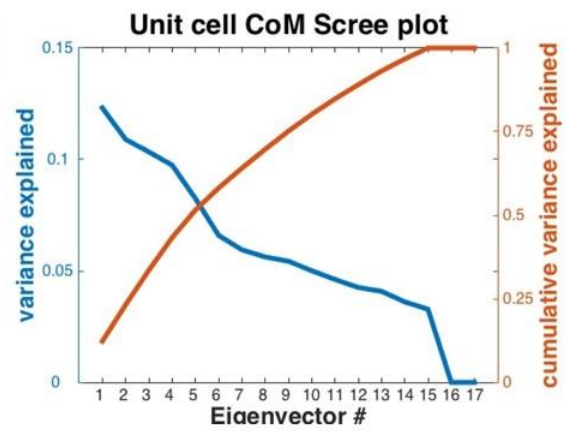

Figure 1. (a) Annular dark-field (ADF) Z-contrast STEM image of $\alpha-\mathrm{RuCl}_{3}$ film (raw data). Inset shows filtered data, where brown and blue crosses correspond to the centers of $\mathrm{Ru} / \mathrm{Cl}$ columns and $\mathrm{Cl}$ columns, respectively. (b) PCA analysis of normal modes of displacement from average unit cell structure. Coloring scheme: first six displacement modes are described by blue, red, green, orange, black, and yellow arrows, respectively. The length of the arrows is magnified by a factor of 20. (c) PCA scree plots.

(a)

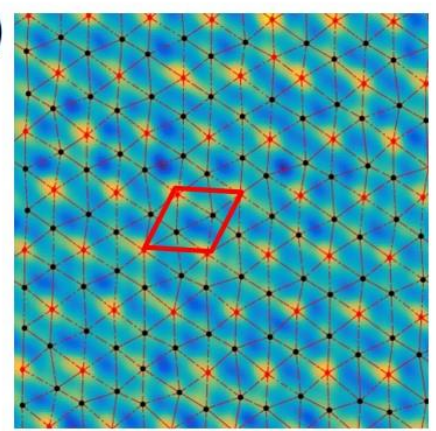

(b)

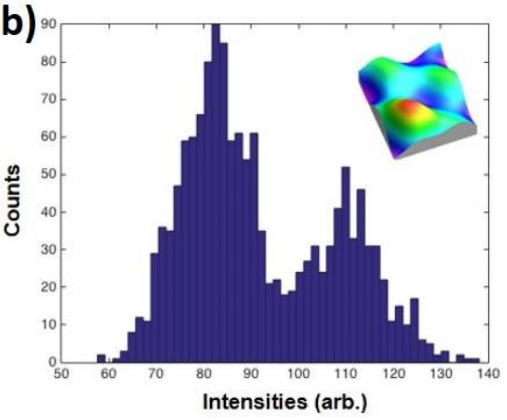

(c)

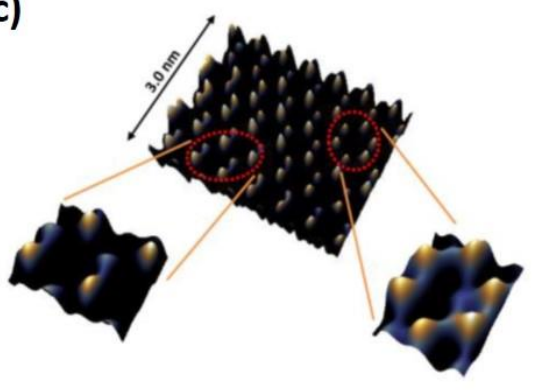

Figure 2. (a) Color-coded lattice overlay of STM image, in which the brightest spots (red dots) form a superlattice of a $\sqrt{3} \times \sqrt{3} R 30^{\circ}$ periodicity (denoted by red rhombus). (b) Histogram-based visualization of distribution of STM intensities at $\mathrm{Cl}$ atoms extracted from $10 \times 10 \mathrm{~nm}^{2}$ surface STM image. (c) Coexistence of rhombic and honeycomb electronic superlattices. 